\title{
Several Properties of Discrete Orlicz Spaces
}

\author{
Pradipta Swiantana Prayoga ${ }^{1}$, Siti Fatimah ${ }^{2}$, and Al Azhary Masta ${ }^{3}$ \\ \{pradiptaenix@gmail.com ${ }^{1}$, sitifatimah@upi.edu ${ }^{2}$, alazhari.masta@upi.edu ${ }^{3}$ \} \\ Department of Mathematics Education, Universitas Pendidikan Indonesia, J1. Dr. Setiabudi 229, \\ Bandung 40154, Indonesia ${ }^{1,2,3}$
}

\begin{abstract}
Introduced by Z. W. Birnbaum and W. Orlicz in 1931, Orlicz spaces are a generalization of the Lebesgue spaces. There are two kinds version of Orlicz spaces which are continuous Orlicz spaces denoted by $L_{\Phi}$ and discrete Orlicz spaces denoted by $\ell_{\Phi}$. The properties of $L_{\Phi}$ spaces are already known. The aim of this study is to discuss the properties of discrete Orlicz spaces. We will see that the properties of continuous Orlicz spaces are also valid in discrete Orlicz spaces. Methods in this research are by using the properties of Young function and Luxemburg Norm. The result of this research is to show that several properties of continuous Orlicz space are also valid in discrete Orlicz spaces.
\end{abstract}

Keywords: Lebesgue Spaces, Continuous Orlicz Spaces, Discrete Orlicz Spaces

\section{Introduction}

Introduced by Z. W. Birnbaum and W. Orlicz in 1931, Orlicz spaces are a generalization of the Lebesgue spaces [1-4]. Many authors have discussed about Orlicz spaces [3-9]. In this paper, we are interested in studying the properties of discrete Orlicz spaces.

First, let us recall the definition of the Young function. A function $\Phi:[0, \infty) \rightarrow[0, \infty)$ is called Young function if $\Phi$ is a convex, continuous, $\lim _{t \rightarrow 0} \Phi(t)=0=\Phi(0)$, and $\lim _{t \rightarrow \infty} \Phi(t)=\infty$. For $\Phi$ is a Young function, we define $\Phi^{-1}(s):=\inf \{r \geq 0: \Phi(r)>s\}$ for every $s \geq 0$.

For $\Phi$ is a Young function, the continuous Orlicz spaces $L_{\Phi}\left(\mathbb{R}^{n}\right)$ is set of measurable functions $f: \mathbb{R}^{n} \rightarrow \mathbb{R}$ such that

$$
\|f\|_{L_{\Phi}\left(\mathbb{R}^{n}\right)}:=\inf \left\{b>0: \int_{\mathbb{R}^{n}} \Phi\left(\frac{|f(x)|}{b}\right) d x \leq 1\right\}
$$

is finite [6]. Furthermore, space $L_{\phi}\left(\mathbb{R}^{n}\right)$ is a Banach space $[2,10,11]$. Sometimes, other researchers also defined the continuous Orlicz spaces as follows:

$1 \quad L_{\Phi}\left(\mathbb{R}^{n}\right)$ is a set of measurable functions $f: \mathbb{R}^{n} \rightarrow \mathbb{R}$ such that

$$
\int_{\mathbb{R}^{n}} \Phi(\alpha|f(x)|) d x<\infty
$$

for some $\alpha>0[8,11]$.

$2 \boldsymbol{L}_{\boldsymbol{\phi}}\left(\mathbb{R}^{n}\right)$ is a set of measurable functions $\boldsymbol{f}: \mathbb{R}^{n} \rightarrow \mathbb{R}$ such that 


$$
\int_{\mathbb{R}^{n}} \Phi\left(\frac{|f(x)|}{\alpha}\right) d x \leq 1
$$

for some $\alpha>0$ [4].

Note that, we can prove that all definitions of continuous Orlicz spaces are equivalent. Furthermore, if $\Phi(t):=t^{p}$ for some $p \geq 1$, then $L_{\phi}\left(\mathbb{R}^{n}\right)=L_{p}\left(\mathbb{R}^{n}\right)$, the Lebesgue spaces of $p$ the integrable functions on $\mathbb{R}^{n}$ [11]. So we can view the continuous Orlicz spaces as a generalization of the Lebesgue spaces.

Now, we recall the definition of discrete Orlicz spaces. Let $\Phi$ be a Young function. The discrete Orlicz spaces $\ell_{\Phi}\left(\mathbb{Z}^{n}\right)$ is the set of all sequences $X:=\left(x_{n}\right): \mathbb{Z}^{n} \rightarrow \mathbb{R}$ such that

$$
\|X\|_{\ell_{\Phi}\left(\mathbb{Z}^{n}\right)}=\inf \left\{b>0: \sum_{k=1}^{\infty} \Phi\left(\frac{\left|x_{k}\right|}{b}\right) \leq 1\right\}<\infty .
$$

In this paper, we denote $A:=\left\{b>0: \sum_{k=1}^{\infty} \Phi\left(\frac{\left|x_{k}\right|}{b}\right) \leq 1\right\}$. So, we have $\|X\|_{\ell_{\Phi}\left(\mathbb{Z}^{n}\right)}=\inf A$. Note that, if $\Phi(t)=t^{p}$, then we have $\ell_{\Phi}\left(\mathbb{Z}^{n}\right)=\ell_{p}\left(\mathbb{Z}^{n}\right)$. The discussion about Lebesgue spaces can be found in $[12,13]$.

In 1989, Maligranda discussed the properties of Orlicz spaces [9]. Later in 2016, Masta $e t$ al. obtained sufficient and necessary conditions for inclusion relation between two Orlicz spaces and between two weak Orlicz spaces by using a different technique from Maligranda [6]. Moreover, they have found that two Orlicz spaces and two weak Orlicz spaces can be compared with respect to Young functions for any measurable set, although the Lebesgue space $L_{p}$ are not comparable with respect to the number $p$. Similar results about the inclusion properties of Orlicz spaces can be found in $[14,15]$. Some properties of continuous Orlicz spaces, we gave as follows.

Lemma 1.1. [2, 6] Let $\Phi$ be a Young function and $f \in L_{\Phi}\left(\mathbb{R}^{n}\right)$. If $0<\|f\|_{L_{\Phi}\left(\mathbb{R}^{n}\right)}<\infty$, then

$$
\int_{\mathbb{R}^{n}} \Phi\left(\frac{|f(x)|}{\|f\|_{\Phi\left(\mathbb{R}^{n}\right)}}\right) d x \leq 1
$$

Furthermore, $\|f\|_{L_{\Phi}\left(\mathbb{R}^{n}\right)} \leq 1$ if only if $\int_{\mathbb{R}^{n}} \Phi\left(\frac{|f(x)|}{\|f\|_{\Phi\left(\mathbb{R}^{n}\right)}}\right) d x \leq 1$.

Lemma 1.2. [2] Let $\Phi$ be a Young function and $f \in L_{\Phi}\left(\mathbb{R}^{n}\right)$. Then the following statements are equivalent.

(1) $\int_{\mathbb{R}^{n}} \Phi\left(\frac{|f(x)|}{\epsilon}\right) d x \leq 1$ for every $\epsilon>0$.

(2) $\|f\|_{L_{\Phi}\left(\mathbb{R}^{n}\right)}=0$.

Lemma 1.3. [2] Let $\Phi$ be a Young function and $f \in L_{\Phi}\left(\mathbb{R}^{n}\right)$. Then the following statements are equivalent.

(1) $\int_{\mathbb{R}^{n}} \Phi(\alpha|f(x)|) d x=0$ for every $\alpha>0$.

(2) $\|f\|_{L_{\Phi}\left(\mathbb{R}^{n}\right)}=0$

Lemma 1.4. [6] Let $\Phi_{1}, \Phi_{2}$ be Young functions and some $C>0$. Then the following statements are equivalent.

(1) $\Phi_{1}(t) \leq \Phi_{2}(C t)$ for every $t>0$. 
(2) For every $f \in L_{\Phi_{2}}\left(\mathbb{R}^{n}\right)$, we have $L_{\Phi_{2}}\left(\mathbb{R}^{n}\right) \subseteq L_{\Phi_{1}}\left(\mathbb{R}^{n}\right)$ with $\|f\|_{L_{\Phi_{1}}\left(\mathbb{R}^{n}\right)} \leq$ $C\|f\|_{L_{\Phi_{2}}\left(\mathbb{R}^{n}\right)}$.

In this paper, we are interested in studying the properties of discrete Orlicz spaces. In connection with Lemmas 1.1, 1.2, 1.3, and 1.4, we shall prove the properties of discrete Orlicz spaces respect to Young function $\Phi$. To obtain our result, we will use the properties of the Young function and Luxemburg Norm [2].

\section{Results}

Now, we have already known some properties of continuous Orlicz spaces. For getting the result, we will apply these properties to discrete Orlicz spaces. First, we present several lemmas in the following. We will also prove that the mapping on discrete Orlicz spaces defines a norm on $\ell_{\Phi}\left(\mathbb{Z}^{n}\right)$.

Lemma 2.1. If $X:=\left(x_{k}\right) \in \ell_{\Phi}\left(\mathbb{Z}^{n}\right)$ and $\|X\|_{\ell_{\Phi}\left(\mathbb{Z}^{n}\right)} \neq 0$, then $\sum_{k=1}^{\infty} \Phi\left(\frac{\left|x_{k}\right|}{\|X\|_{\ell_{\Phi}\left(\mathbb{Z}^{n}\right)}}\right) \leq 1$.

Proof.

Let $X:=\left(x_{k}\right) \in \ell_{\Phi}\left(\mathbb{Z}^{n}\right)$ such that $\|X\|_{\ell_{\Phi}\left(\mathbb{Z}^{n}\right)} \neq 0$ and take an arbitrary $\epsilon>0$, note that $\|X\|_{\ell_{\Phi}\left(\mathbb{Z}^{n}\right)}+\epsilon$ is not a lower bound of the set $A:=\left\{b>0: \sum_{k=1}^{\infty} \Phi\left(\frac{\left|x_{k}\right|}{b}\right) \leq 1\right\}$, then there exists $b_{1} \in A$ such that $b_{1} \leq\|X\|_{\ell_{\Phi}\left(\mathbb{Z}^{n}\right)}+\epsilon$. Note that, $b_{1} \leq\|X\|_{\ell_{\Phi}\left(\mathbb{Z}^{n}\right)}+\epsilon$ are equivalent to $\frac{1}{\|X\|_{\ell_{\Phi}\left(\mathbb{Z}^{n}\right)+\epsilon}} \leq \frac{1}{b_{1}}$. Since $\left|x_{k}\right|$ is nonnegative for every $k \in \mathbb{N}$, we have

$$
\frac{\left|x_{k}\right|}{\|X\|_{\ell_{\Phi}\left(\mathbb{Z}^{n}\right)}+\epsilon} \leq \frac{\left|x_{k}\right|}{b_{1}} .
$$

Because $\Phi$ is increasing, we have $\Phi\left(\frac{\left|x_{k}\right|}{\|X\|_{\ell_{\Phi}\left(\mathbb{Z}^{n}\right)}+\epsilon}\right) \leq \Phi\left(\frac{\left|x_{k}\right|}{b_{1}}\right) \leq 1$ for every $k \in \mathbb{N}$. So we obtain equation 7.

$$
\sum_{k=1}^{\infty} \Phi\left(\frac{\left|x_{k}\right|}{\|X\|_{\ell_{\Phi}\left(\mathbb{Z}^{n}\right)}+\epsilon}\right) \leq \sum_{k=1}^{\infty} \Phi\left(\frac{\left|x_{k}\right|}{b_{1}}\right) \leq 1
$$

Since the following inequality $\sum_{k=1}^{\infty} \Phi\left(\frac{\left|x_{k}\right|}{\|X\|_{\ell_{\Phi}\left(\mathbb{Z}^{n}\right)}+\epsilon}\right) \leq 1$ is true for $\epsilon>0$, we can conclude that

$$
\sum_{k=1}^{\infty} \Phi\left(\frac{\left|x_{k}\right|}{\|X\|_{\ell_{\Phi}\left(\mathbb{Z}^{n}\right)}}\right) \leq 1,
$$

as desired.

Lemma 2.2. Let $\Phi$ be a Young function. Then the following statements are equivalent.

(1) $\sum_{k=1}^{\infty} \Phi\left(\left|x_{k}\right|\right) \leq 1$

(2) $\|X\|_{\ell_{\Phi}\left(\mathbb{Z}^{n}\right)} \leq 1$ for every $X:=\left(x_{k}\right) \in \ell_{\Phi}\left(\mathbb{Z}^{n}\right)$.

Proof. 
Suppose that $(a)$ holds, then we have $\sum_{k=1}^{\infty} \Phi\left(\left|x_{k}\right|\right)=\sum_{k=1}^{\infty} \Phi\left(\frac{\left|x_{k}\right|}{1}\right) \leq 1$. Therefore, $1 \in A:=$ $\left\{b>0: \sum_{k=1}^{\infty} \Phi\left(\frac{\left|x_{k}\right|}{b}\right) \leq 1\right\}$. So, $\|X\|_{\ell_{\Phi}\left(\mathbb{Z}^{n}\right)} \leq 1$. Now, suppose that (b) holds, then we have $\|X\|_{\ell_{\Phi}\left(\mathbb{Z}^{n}\right)} \leq 1$. Hence, we have equation 9

$$
\left|x_{k}\right| \leq \frac{\left|x_{k}\right|}{\|X\|_{\ell_{\Phi}\left(\mathbb{Z}^{n}\right)}} \text {. }
$$

Because $\Phi$ is increasing, then we obtain equation 10

$$
\sum_{k=1}^{\infty} \Phi\left(\left|x_{k}\right|\right) \leq \sum_{k=1}^{\infty} \Phi\left(\frac{\left|x_{k}\right|}{\|X\|_{\ell_{\Phi}\left(\mathbb{Z}^{n}\right)}}\right) \leq 1 .
$$

Hence, we can conclude that

$$
\sum_{k=1}^{\infty} \Phi\left(\left|x_{k}\right|\right) \leq 1
$$

Lemma 2.3. Let $\Phi$ be a Young function. Then the following statements are equivalent.

(1) $\sum_{k=1}^{\infty} \Phi\left(\frac{\left|x_{k}\right|}{\epsilon}\right) \leq 1$ for every $\epsilon>0$.

(2) $\|X\|_{\ell_{\Phi}\left(\mathbb{Z}^{n}\right)}=0$ for every $X:=\left(x_{k}\right) \in \ell_{\Phi}\left(\mathbb{Z}^{n}\right)$.

Proof.

Suppose that ( $a$ ) holds, then $\epsilon \in A:=\left\{b>0: \sum_{k=1}^{\infty} \Phi\left(\frac{\left|x_{k}\right|}{b}\right) \leq 1\right\}$ for every $\epsilon>0$. Therefore, $0 \leq\|X\|_{\ell_{\Phi}\left(\mathbb{Z}^{n}\right)} \leq \epsilon$ for any $\epsilon>0$. Consequently, we can conclude that $\|X\|_{\ell_{\Phi}\left(\mathbb{Z}^{n}\right)}=0$. Now, suppose that (b) holds. By contradiction, there exist $\epsilon_{0}>0$ such that $\sum_{k=1}^{\infty} \Phi\left(\frac{\left|x_{k}\right|}{\epsilon_{0}}\right)>1$, hence $\epsilon_{0} \notin A$. Take an arbitrary $b_{1} \in A$, then we have $b_{1} \neq \epsilon_{0}$. Therefore, $\|X\|_{\ell_{\Phi}\left(\mathbb{Z}^{n}\right)} \leq b_{1}$. Next, we consider two cases.

Case 1: if $b_{1}<\epsilon_{0}$, then we have $\frac{1}{\epsilon_{0}}<\frac{1}{b_{1}}$. Since $\left|x_{k}\right|$ is nonnegative for every $k \in \mathbb{N}$, we have $\frac{\left|x_{k}\right|}{\epsilon_{0}}<\frac{\left|x_{k}\right|}{b_{1}}$. Hence, we have equation 12

$$
\sum_{k=1}^{\infty} \Phi\left(\frac{\left|x_{k}\right|}{\epsilon_{0}}\right)<\sum_{k=1}^{\infty} \Phi\left(\frac{\left|x_{k}\right|}{b_{1}}\right) \leq \sum_{k=1}^{\infty} \Phi\left(\frac{\left|x_{k}\right|}{b}\right) \leq 1 .
$$

Observe equation 13 ,

$$
\sum_{k=1}^{\infty} \Phi\left(\frac{\left|x_{k}\right|}{\epsilon_{0}}\right) \leq \sum_{k=1}^{\infty} \Phi\left(\frac{\left|x_{k}\right|}{b_{1}}\right) \leq 1 .
$$

Hence, we have $\|X\|_{\ell_{\Phi}\left(\mathbb{Z}^{n}\right)} \leq 1$. This contradicts with the fact that $\|X\|_{\ell_{\Phi}\left(\mathbb{Z}^{n}\right)}=0$. 
Case 2: if $b_{1}>\epsilon_{0}$, then we have $\|X\|_{\ell_{\Phi}\left(\mathbb{Z}^{n}\right)} \geq \epsilon_{0}>0$ or we can see that $0<\|X\|_{\ell_{\Phi}\left(\mathbb{Z}^{n}\right)}$. This contradicts with the fact that $\|X\|_{\ell_{\Phi}\left(\mathbb{Z}^{n}\right)}=0$.

From case 1 and 2, we conclude that $\sum_{k=1}^{\infty} \Phi\left(\frac{\left|x_{k}\right|}{\epsilon}\right) \leq 1$ for every $\epsilon>0$.

Lemma 2.4. Let $\Phi$ be a Young function. Then the following statements are equivalent.

(1) $\sum_{k=1}^{\infty} \Phi\left(\alpha\left|x_{k}\right|\right)=0$ for every $\alpha>0$.

Proof.

(2) $\|X\|_{\ell_{\Phi}\left(\mathbb{Z}^{n}\right)}=0$ for every $X:=\left(x_{k}\right) \in \ell_{\Phi}\left(\mathbb{Z}^{n}\right)$.

Suppose that (a) holds. Then $\sum_{k=1}^{\infty} \Phi\left(\alpha\left|x_{k}\right|\right)=0 \leq 1$, then $\sum_{k=1}^{\infty} \Phi\left(\frac{\left|x_{k}\right|}{\frac{1}{\alpha}}\right)=0 \leq 1$. Hence, we can obtain $0 \leq\|X\|_{\ell_{\Phi}\left(\mathbb{Z}^{n}\right)} \leq \frac{1}{\alpha}$ for any $\alpha>0$. So we can conclude that $\|X\|_{\ell_{\Phi}\left(\mathbb{Z}^{n}\right)}=0$. Now, suppose that $(b)$ holds. Observe that $\sum_{k=1}^{\infty} \Phi\left(\alpha\left|x_{k}\right|\right)=\sum_{k=1}^{\infty} \Phi\left(\epsilon \frac{\alpha\left|x_{k}\right|}{\epsilon}\right)$. Because $\Phi$ is a convex function, we have

$$
\begin{aligned}
\sum_{k=1}^{\infty} \Phi\left(\epsilon \frac{\alpha\left|x_{k}\right|}{\epsilon}\right) & \leq \epsilon \sum_{k=1}^{\infty} \Phi\left(\frac{\alpha\left|x_{k}\right|}{\epsilon}\right) \\
& =\epsilon \sum_{k=1}^{\infty} \Phi\left(\frac{\left|x_{k}\right|}{\frac{\epsilon}{\alpha}}\right)
\end{aligned}
$$$$
\leq \epsilon
$$

Therefore, $0 \leq \sum_{k=1}^{\infty} \Phi\left(\alpha\left|x_{k}\right|\right) \leq \epsilon$. So, we can conclude that $\sum_{k=1}^{\infty} \Phi\left(\alpha\left|x_{k}\right|\right)=0$.

Theorem 2.5. Let $\Phi$ be a Young function. The mapping $\|\cdot\|_{\ell_{\Phi}\left(\mathbb{Z}^{n}\right)}$ defines a norm on $\ell_{\Phi}\left(\mathbb{Z}^{n}\right)$. Proof.

Since $\|X\|_{\ell_{\Phi}\left(\mathbb{Z}^{n}\right)}=\inf \left\{b>0: \sum_{k=1}^{\infty} \Phi\left(\frac{\left|x_{k}\right|}{b}\right) \leq 1\right\}$, by definition of infimum, we have $\|X\|_{\ell_{\Phi}\left(\mathbb{Z}^{n}\right)} \geq 0$. Now, we will prove that $\|X\|_{\ell_{\Phi}\left(\mathbb{Z}^{n}\right)}=0$ if and only if $X=0$ for every $X:=$ $x_{k} \in \ell_{\Phi}\left(\mathbb{Z}^{n}\right)$ and $k \in \mathbb{N}$. If $X=0$, then $x_{k}=0$ for every $k \in \mathbb{N}$. Now, take an arbitrary $b>0$. So, we have $\frac{\left|x_{k}\right|}{b}=0$. Because $\Phi$ is increasing, we have $\Phi\left(\frac{\left|x_{k}\right|}{b}\right)=\Phi(0)=0$. Hence, $\sum_{k=1}^{\infty} \Phi\left(\frac{\left|x_{k}\right|}{b}\right)=0$. By the definition of norm, we have $\|X\|_{\ell_{\Phi}\left(\mathbb{Z}^{n}\right)} \leq b$ for every $b \geq 0$. Consequently, we have $\|X\|_{\ell_{\Phi}\left(\mathbb{Z}^{n}\right)}=0$. Now, let $\|X\|_{\ell_{\Phi}\left(\mathbb{Z}^{n}\right)}=0$. By Lemma 2.4, we have $\sum_{k=1}^{\infty} \Phi\left(\alpha\left|x_{k}\right|\right)=0$ for $\alpha>0$. Since $\Phi$ is a non-negative function, then $\Phi\left(\alpha\left|x_{k}\right|\right)=0$, for every $k \in \mathbb{N}$. We know that $\Phi\left(\alpha\left|x_{k}\right|\right)=0$ when $\alpha\left|x_{k}\right|=0$. Since $\alpha>0$, then $x_{k}=0$ for every $k \in \mathbb{N}$. So we can conclude that $X=0$. Now, we will prove that $\|\alpha X\|_{\ell_{\Phi}\left(\mathbb{Z}^{n}\right)}=|\alpha|\|X\|_{\ell_{\Phi}\left(\mathbb{Z}^{n}\right)}$ for every $X:=x_{k} \in \ell_{\Phi}\left(\mathbb{Z}^{n}\right), k \in \mathbb{N}$, and $\alpha \in \mathbb{R}$. We consider two cases.

Case 1: for $\alpha \neq 0$ (setting $c=\frac{b}{|\alpha|}$ ). Observe equation 15 .

$$
\|\alpha X\|_{\ell_{\Phi}\left(\mathbb{Z}^{n}\right)}=\inf \left\{b>0: \sum_{k=1}^{\infty} \Phi\left(\frac{\left|\alpha x_{k}\right|}{b}\right) \leq 1\right\}
$$




$$
\begin{aligned}
& =\inf \left\{b>0: \sum_{k=1}^{\infty} \Phi\left(\frac{\left|x_{k}\right|}{\frac{b}{|\alpha|}}\right) \leq 1\right\} \\
& =\inf \left\{c|\alpha|>0: \sum_{k=1}^{\infty} \Phi\left(\frac{\left|x_{k}\right|}{c}\right) \leq 1\right\} \\
& =|\alpha| \inf \left\{c>0: \sum_{k=1}^{\infty} \Phi\left(\frac{\left|x_{k}\right|}{c}\right) \leq 1\right\} \\
& =|\alpha|\|X\|_{\ell_{\Phi}\left(\mathbb{Z}^{n}\right) .}
\end{aligned}
$$

Case 2: for $\alpha=0$. Observe that for every $X:=x_{k} \in \ell_{\Phi}\left(\mathbb{Z}^{n}\right)$, we have equation 16

$$
\|\alpha X\|_{\ell_{\Phi}\left(\mathbb{Z}^{n}\right)}=\|0\|_{\ell_{\Phi}\left(\mathbb{Z}^{n}\right)}=0=0\|X\|_{\ell_{\Phi}\left(\mathbb{Z}^{n}\right)}=\alpha\|X\|_{\ell_{\Phi}\left(\mathbb{Z}^{n}\right)} .
$$

Hence, from case 1 and 2, we can conclude that $\|\alpha X\|_{\ell_{\Phi}\left(\mathbb{Z}^{n}\right)}=|\alpha|\|X\|_{\ell_{\Phi}\left(\mathbb{Z}^{n}\right)}$ for every $X:=$ $x_{k} \in \ell_{\Phi}\left(\mathbb{Z}^{n}\right), k \in \mathbb{N}$, and $\alpha \in \mathbb{R}$.

Next, we will prove that $\left\|X_{1}+X_{2}\right\|_{\ell_{\Phi}\left(\mathbb{Z}^{n}\right)} \leq\left\|X_{1}\right\|_{\ell_{\Phi}\left(\mathbb{Z}^{n}\right)}+\left\|X_{2}\right\|_{\ell_{\Phi}\left(\mathbb{Z}^{n}\right)}$, for every $X_{1}, X_{2} \in$ $\ell_{\Phi}\left(\mathbb{Z}^{n}\right)$ with $X_{1}:=x_{k_{1}}, X_{2}:=x_{k_{2}}$, and $k \in \mathbb{N}$.

Let $X_{1}, X_{2} \in \ell_{\Phi}\left(\mathbb{Z}^{n}\right)$ with $X_{1}:=x_{k_{1}}, X_{2}:=x_{k_{2}}$ for every $k \in \mathbb{N}$. So, we have

$$
\begin{aligned}
& \sum_{k=1}^{\infty} \Phi\left(\frac{\left|x_{k_{1}}+x_{k_{2}}\right|_{\ell_{\Phi}\left(\mathbb{Z}^{n}\right)}}{\left\|X_{1}\right\|_{\ell_{\Phi}\left(\mathbb{Z}^{n}\right)}+\left\|X_{2}\right\|_{\ell_{\Phi}\left(\mathbb{Z}^{n}\right)}}\right) \\
& \leq \sum_{k=1}^{\infty} \Phi\left(\frac{\left|x_{k_{1}}\right|+\left|x_{k_{2}}\right|}{\left\|X_{1}\right\|_{\ell_{\Phi}\left(\mathbb{Z}^{n}\right)}+\left\|X_{2}\right\|_{\ell_{\Phi}\left(\mathbb{Z}^{n}\right)}}\right)
\end{aligned}
$$$$
=\sum_{k_{\infty}=1}^{\infty} \Phi\left(\frac{\left|x_{k_{1}}\right|}{\left\|X_{1}\right\|_{\ell_{\Phi}\left(\mathbb{Z}^{n}\right)}+\left\|X_{2}\right\|_{\ell_{\Phi}\left(\mathbb{Z}^{n}\right)}}+\frac{\left|x_{k_{2}}\right|}{\left\|X_{1}\right\|_{\ell_{\Phi}\left(\mathbb{Z}^{n}\right)}+\left\|X_{2}\right\|_{\ell_{\Phi}\left(\mathbb{Z}^{n}\right)}}\right)
$$$$
=\sum_{k=1}^{\infty} \Phi\left(\frac{\left\|x_{1}\right\|_{\ell_{\Phi}\left(\mathbb{Z}^{n}\right)}}{\left\|X_{1}\right\|_{\ell_{\Phi}\left(\mathbb{Z}^{n}\right)}+\left\|X_{2}\right\|_{\ell_{\Phi}\left(\mathbb{Z}^{n}\right)}} \frac{\left|x_{k_{1}}\right|}{\left\|X_{1}\right\|_{\ell_{\Phi}\left(\mathbb{Z}^{n}\right)}}+\frac{\left\|x_{2}\right\|_{\ell_{\Phi}\left(\mathbb{Z}^{n}\right)}}{\left\|X_{1}\right\|_{\ell_{\Phi}\left(\mathbb{Z}^{n}\right)}+\left\|X_{2}\right\|_{\ell_{\Phi}\left(\mathbb{Z}^{n}\right)}} \frac{\left|x_{k_{2}}\right|}{\left\|X_{2}\right\|_{\ell_{\Phi}\left(\mathbb{Z}^{n}\right)}}\right)
$$

$$
\begin{aligned}
\leq \frac{\left\|X_{1}\right\|_{\ell_{\Phi}\left(\mathbb{Z}^{n}\right)}}{\left\|X_{1}\right\|_{\ell_{\Phi}\left(\mathbb{Z}^{n}\right)}+\left\|X_{2}\right\|_{\ell_{\Phi}\left(\mathbb{Z}^{n}\right)}} \sum_{k=1}^{\infty} \Phi\left(\frac{\left|x_{k_{1}}\right|}{\left\|X_{1}\right\|_{\ell_{\Phi}\left(\mathbb{Z}^{n}\right)}}\right) \\
\quad+\frac{\left\|X_{2}\right\|_{\ell_{\Phi}\left(\mathbb{Z}^{n}\right)}}{\left\|X_{1}\right\|_{\ell_{\Phi}\left(\mathbb{Z}^{n}\right)}+\left\|X_{2}\right\|_{\ell_{\Phi}\left(\mathbb{Z}^{n}\right)}} \sum_{k=1}^{\infty} \Phi\left(\frac{\left|x_{k_{2}}\right|}{\left\|X_{2}\right\|_{\ell_{\Phi}\left(\mathbb{Z}^{n}\right)}}\right)
\end{aligned}
$$

$\leq \frac{\left\|X_{1}\right\|_{\ell_{\Phi}\left(\mathbb{Z}^{n}\right)}}{\left\|X_{1}\right\|_{\ell_{\Phi}\left(\mathbb{Z}^{n}\right)}+\left\|X_{2}\right\|_{\ell_{\Phi}\left(\mathbb{Z}^{n}\right)}}+\frac{\left\|X_{2}\right\|_{\ell_{\Phi}\left(\mathbb{Z}^{n}\right)}}{\left\|X_{1}\right\|_{\ell_{\Phi}\left(\mathbb{Z}^{n}\right)}+\left\|X_{2}\right\|_{\ell_{\Phi}\left(\mathbb{Z}^{n}\right)}}$

$=\frac{\left\|X_{1}\right\|_{\ell_{\Phi}\left(\mathbb{Z}^{n}\right)}+\left\|X_{2}\right\|_{\ell_{\Phi}\left(\mathbb{Z}^{n}\right)}}{\left\|X_{1}\right\|_{\ell_{\Phi}\left(\mathbb{Z}^{n}\right)}+\left\|X_{2}\right\|_{\ell_{\Phi}\left(\mathbb{Z}^{n}\right)}}$

$=1$.

By definition of $\left\|X_{1}+X_{2}\right\|_{\ell_{\Phi}\left(\mathbb{Z}^{n}\right)}$ for every $X_{1}, X_{2} \in \ell_{\Phi}\left(\mathbb{Z}^{n}\right)$ with $X_{1}:=x_{k_{1}}, X_{2}:=x_{k_{2}}$, and $k \in$ $\mathbb{N}$, we have equation 17 


$$
\left\|x_{1}+x_{2}\right\|_{\ell_{\Phi}\left(\mathbb{Z}^{n}\right)} \leq\left\|x_{1}\right\|_{\ell_{\Phi}\left(\mathbb{Z}^{n}\right)}+\left\|x_{2}\right\|_{\ell_{\Phi}\left(\mathbb{Z}^{n}\right)} .
$$

So, the mapping $\|\cdot\|_{\ell_{\Phi}\left(\mathbb{Z}^{n}\right)}$ defines a norm on $\ell_{\Phi}\left(\mathbb{Z}^{n}\right)$.

Theorem 2.6. Let $\Phi_{1}, \Phi_{2}$ be Young functions. If $\Phi_{1}(t) \leq C \Phi_{2}(t)$ for every t $>0$ and some $C>0$, then $\|X\|_{\ell_{\Phi_{1}}\left(\mathbb{Z}^{n}\right)} \leq \max \{1, C\}\|X\|_{\ell_{\Phi_{2}}\left(\mathbb{Z}^{n}\right)}$ for every $X:=\left(x_{k}\right) \in \ell_{\Phi}\left(\mathbb{Z}^{n}\right)$.

Proof.

Let $A_{1}:=\left\{b>0: \sum_{k=1}^{\infty} \Phi_{1}\left(\frac{\left|x_{k}\right|}{b}\right) \leq 1\right\}$ and $A_{2}:=\left\{b>0: \sum_{k=1}^{\infty} \Phi_{2}\left(\frac{\left|x_{k}\right|}{b}\right) \leq 1\right\}$. Hence, we have $\|X\|_{\ell_{\Phi_{1}}\left(\mathbb{Z}^{n}\right)}=\inf A_{1}$ and $\|X\|_{\ell_{\Phi_{2}}\left(\mathbb{Z}^{n}\right)}=\inf A_{2}$. Because $\Phi_{1}\left(\frac{\left|x_{k}\right|}{b}\right) \leq C \Phi_{2}\left(\frac{\left|x_{k}\right|}{b}\right)$ is true for every $k \in \mathbb{N}$, then we have equation 18

$$
\sum_{k=1}^{\infty} \Phi_{1}\left(\frac{\left|x_{k}\right|}{b}\right) \leq C \sum_{k=1}^{\infty} \Phi_{2}\left(\frac{\left|x_{k}\right|}{b}\right)
$$

Now, we consider two cases.

Case 1: for $0<C \leq 1$. Observe equation 19

$$
\sum_{k=1}^{\infty} \Phi_{1}\left(\frac{\left|x_{k}\right|}{b}\right) \leq \sum_{k=1}^{\infty} \Phi_{2}\left(\frac{\left|x_{k}\right|}{b}\right) \leq 1
$$

Since $b \in A_{2}$ implies $b \in A_{1}$, so we have $A_{2} \subseteq A_{1}$. Thus, $\inf A_{1} \leq \inf A_{2}$. So, we have

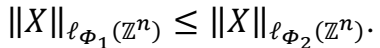

Case 2: for $C>1$. Observe equation 20

$$
\sum_{k=1}^{\infty} \Phi_{1}\left(\frac{\left|x_{k}\right|}{b}\right) \leq C \sum_{k=1}^{\infty} \Phi_{2}\left(\frac{\left|x_{k}\right|}{b}\right) .
$$

Therefore to be equation 21

$$
\sum_{k=1}^{\infty} \Phi_{1}\left(\frac{\frac{1}{C}\left|x_{k}\right|}{b}\right) \leq \frac{1}{C} \sum_{k=1}^{\infty} \Phi_{1}\left(\frac{\left|x_{k}\right|}{b}\right) \leq \sum_{k=1}^{\infty} \Phi_{2}\left(\frac{\left|x_{k}\right|}{b}\right) \leq 1 .
$$

Note that for $b \in A_{2}$ imply to equation 22

$$
\sum_{k=1}^{\infty} \Phi_{1}\left(\frac{\frac{1}{C}\left|x_{k}\right|}{b}\right) \leq 1
$$


Now, let $A_{3}:=\left\{b>0: \sum_{k=1}^{\infty} \Phi_{1}\left(\frac{\frac{1}{c}\left|x_{k}\right|}{b}\right) \leq 1\right\}$. Hence, $b \in A_{2}$ implies $b \in A_{3}$. Because $\inf A_{3} \leq$ $\inf A_{2}$, we have $\left\|\frac{1}{c} X\right\|_{\ell_{\Phi_{1}\left(\mathbb{Z}^{n}\right)}} \leq\|X\|_{\ell_{\Phi_{2}}\left(\mathbb{Z}^{n}\right)}$. Because $c>1$, we have $\frac{1}{c}\|X\|_{\left.\ell_{\Phi_{1}} \mathbb{Z}^{n}\right)} \leq$ $\|X\|_{\ell_{\Phi_{2}}\left(\mathbb{Z}^{n}\right)}$. Consequently, we have $\|X\|_{\ell_{\Phi_{1}}\left(\mathbb{Z}^{n}\right)} \leq C\|X\|_{\ell_{\Phi_{2}}\left(\mathbb{Z}^{n}\right)}$.

So, we can conclude that for $C>0$, if $\Phi_{1}\left(\frac{\left|x_{k}\right|}{b}\right) \leq C \Phi_{2}\left(\frac{\left|x_{k}\right|}{b}\right)$, then we have $\|X\|_{\left.\ell_{\Phi_{1}} \mathbb{Z}^{n}\right)} \leq$ $D\|X\|_{\ell_{\Phi_{2}}\left(\mathbb{Z}^{n}\right)}$, with $D:=\max \{1, C\}$ for every $X:=\left(x_{k}\right) \in \ell_{\Phi}\left(\mathbb{Z}^{n}\right)$.

Theorem 2.7. Let $\Phi_{1}, \Phi_{2}$ be Young functions. If $\Phi_{1}(t) \leq \Phi_{2}(C t)$ for every $t>0$ and some $C>0$, then $\|X\|_{\ell_{\Phi_{1}}\left(\mathbb{Z}^{n}\right)} \leq C\|X\|_{\left.\ell_{\Phi_{2}} \mathbb{Z}^{n}\right)}$ for every $X:=\left(x_{k}\right) \in \ell_{\Phi}\left(\mathbb{Z}^{n}\right)$.

Proof.

Let $A_{1}:=\left\{b>0: \sum_{k=1}^{\infty} \Phi_{1}\left(\frac{\left|x_{k}\right|}{b}\right) \leq 1\right\}$ and $A_{2}:=\left\{b>0: \sum_{k=1}^{\infty} \Phi_{2}\left(\frac{C\left|x_{k}\right|}{b}\right) \leq 1\right\}$. Hence, we have $\|X\|_{\ell_{\Phi_{1}}\left(\mathbb{Z}^{n}\right)}=\inf A_{1}$ and $\|C X\|_{\ell_{\Phi_{2}}\left(\mathbb{Z}^{n}\right)}=\inf A_{2}$. Because $\Phi_{1}\left(\frac{\left|x_{k}\right|}{b}\right) \leq \Phi_{2}\left(\frac{C\left|x_{k}\right|}{b}\right)$ is true for every $k \in \mathbb{N}$, then we have equation 23

$$
\sum_{k=1}^{\infty} \Phi_{1}\left(\frac{\left|x_{k}\right|}{b}\right) \leq \sum_{k=1}^{\infty} \Phi_{2}\left(\frac{C\left|x_{k}\right|}{b}\right) .
$$

If $b \in A_{2}$, then we have equation 24

$$
\sum_{k=1}^{\infty} \Phi_{1}\left(\frac{\left|x_{k}\right|}{b}\right) \leq \sum_{k=1}^{\infty} \Phi_{2}\left(\frac{C\left|x_{k}\right|}{b}\right) \leq 1
$$

which implies $b \in A_{1}$. So we have $A_{2} \subseteq A_{1}$. Thus, $\inf A_{1} \leq \inf A_{2}$. So, we have $\|X\|_{\left.\ell_{\Phi_{1}} \mathbb{Z}^{n}\right)} \leq$

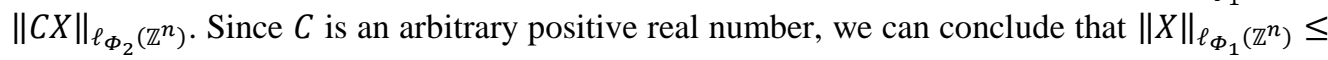
$\|C X\|_{\ell_{\Phi_{2}}\left(\mathbb{Z}^{n}\right)}=C\|X\|_{\ell_{\Phi_{2}}\left(\mathbb{Z}^{n}\right)}$ for every $X:=\left(x_{k}\right) \in \ell_{\Phi}\left(\mathbb{Z}^{n}\right)$ Furthermore, we have $\ell_{\Phi_{2}}\left(\mathbb{Z}^{n}\right) \subseteq$ $\ell_{\Phi_{1}}\left(\mathbb{Z}^{n}\right)$.

\section{Remark.}

Theorems 2.6 and 2.7 are often called as the sufficient condition for inclusion properties of discrete Orlicz spaces. Analog results for other properties of Orlicz spaces can be found in $[1$, $5,6,8,12,14,15,16]$.

\section{Conclusion}

We have shown the properties of discrete Orlicz spaces. The discrete Orlicz spaces are a generalization of $\boldsymbol{p}$-summable sequence spaces in [11]. From Lemmas 2.1, 2.2, 2.3, 2.4, and Theorem 2.5, we can see that several properties of continuous Orlicz spaces are also true for discrete Orlicz spaces. In the proof of our results, we used the properties of Young function and Luxemburg norm in discrete Orlicz spaces. Furthermore, we also obtain sufficient conditions for the inclusion properties of discrete Orlicz spaces as presented in Theorems 2.6 and 2.7. 


\section{Acknowledgment.}

This research is supported by Hibah Penguatan Kompetensi UPI and Hibah Afirmasi dan Pembinaan Dosen UPI 2019.

\section{References}

[1] Orlicz, W.: Linear Functional Analysis. Series in Real Analysis Vol. 4. World Scientific, Singapore (1992)

[2] Léonard, C.: Orlicz Spaces. Research report: http://leonard.perso.math.cnrs.fr/papers/LeonardOrlicz\%20spaces.pdf, accessed in 7 September 2019. pp. 1-10 (2007)

[3] Kufner, A., John, O., and Fucikk, S.: Function Spaces. Noordhoff International Publishing, Czechoslovakia (1977)

[4] Rao, M. M. and Ren Z. D.: Theory of Orlicz spaces. Vol. 146 of Monographs and Textbooks in Pure and Applied Mathematics. Marcel Dekker Inc., New York (1991)

[5] Maligranda, L. and Mastylo, M.: Inclusion Mappings between Orlicz Sequence Spaces. Journal of Functional Analysis. Vol. 176, pp. 264-279 (2000)

[6] Masta, A. A., Gunawan, H., and Setya-Budhi, W.: Inclusion property of Orlicz and weak Orlicz spaces. Journal of Mathematical and Fundamental Sciences Vol. 48-3, pp. 193-203 (2016)

[7] Masta, A. A., Ifronika, and Fatimah, S.: Sufficient and Necessary Conditions for Holder's Inequality in Weighted Orlicz Spaces, to appear in International Journal of Applied Physics and Mathematics (2019)

[8] Osançlıol, A.: A Note On The Definition of An Orlicz Space. Afyon Kocatepe University Journal of Science and Engineering. Vol. 15, pp. 1-6 (2015)

[9] Maligranda, L.: Orlicz Spaces and Interpolation. Departamento de Matemática. Universidade Estadual de Campinas. (1989)

[10] Luxemburg, W. A. J.: Banach Function Spaces. Thesis. Technische Hogeschool te Delft. (1955)

[11] Masta, A. A.: On Uniform Orlicz Spaces. Thesis. Bandung Institute of Technology. (2013)

[12] Masta, A. A., Sumiaty, E., Taqiyuddin, M., and Pradipta, I: The sufficient condition for inclusion properties of discrete weighted Lebesgue spaces. Journal of Physics: Conference Series 1013012152 (2018)

[13] Nekvinda, A.: Embeddings between discrete weighted Lebesgue spaces with variable exponents Mathematical Inequalities and Applications. Vol. 10-1, pp. 165 - 172 (2007)

[14] Masta, A. A., Ifronika, and Taqiyuddin, M.: Inclusion properties of weighted weak Orlicz spaces. Research report: https://arxiv.org/abs/1710.04537, accessed in 12 September 2019. (2017)

[15] Taqiyuddin, M. and Masta, A. A.: Inclusion properties of Orlicz spaces and weak Orlicz spaces generated by concave function. IOP Conference Series: Materials Science and Engineering 288012103. pp. 1-5 (2018)

[16] Masta, A. A., Ifronika, and Taqiyuddin, M.: A Note on Inclusion properties of weighted Orlicz spaces. To appear in Journal of the Indonesian Mathematical Society. (2019) 